\title{
Theoretical and Experimental Understanding of the Anomalous Odd-to-Even Isotope Ratios of Tin in a $1+1$ Single-Colour Resonance Ionization Mass Spectrometry: Revisited
}

\author{
Manda Sankari \\ National Centre for Compositional Characterisation of Materials, \\ Bhabha Atomic Research Centre, Hyderabad 500 062, India \\ Correspondence should be addressed to Manda Sankari, mandasankari@rediffmail.com
}

Received 5 October 2010; Accepted 11 March 2011

Academic Editor: Wonho Jhe

Copyright (C) 2011 Manda Sankari. This is an open access article distributed under the Creative Commons Attribution License, which permits unrestricted use, distribution, and reproduction in any medium, provided the original work is properly cited.

\begin{abstract}
Differences in the odd to even response for tin isotopes has been observed earlier in resonance ionization experiments, resulting in anomalous odd to even isotope ratios. I have used a theoretical approach known as the spectral simulation approach to understand the cause for such anomaly and the anomaly has also been experimentally verified and found to be in good agreement. The effects of laser parameters such as intensity, accuracy of the excitation laser wavelength and bandwidth on the determination of the tin isotope ratio have been analyzed theoretically and experimentally. The source for such anomalies was found to be the inaccuracy in the excitation laser wavelength. For the $5 \mathrm{p}^{2}\left({ }^{3} \mathrm{P}_{0}\right)-5 \mathrm{p} 6 \mathrm{~s}\left({ }^{3} \mathrm{P}_{1}^{0}\right)(286.3317 \mathrm{~nm})$ transition, an inaccuracy of the order of $\gamma_{L}$ in the peak frequency of the excitation laser $\left(\gamma_{L}=15 \mathrm{GHz}\right)$ can cause anomalies as large as $\sim 31 \%(\beta=0.31)$. Use of a very large bandwidth laser $(\sim 60 \mathrm{GHz})$ reduces the anomaly to as small as -0.003 . Alternatively by employing a relatively narrow band laser $(\sim 1.2 \mathrm{GHz})$, it has been observed that inaccuracy of the order of 3-4 $\gamma_{L}$ in the laser peak frequency does not induce anomalies $>0.05$. The isotope ratio is sensitive to the inaccuracy in the excitation laser wavelength for an intermediate linewidth laser.
\end{abstract}

\section{Introduction}

Resonance ionization mass spectrometry (RIMS) has emerged as a powerful analytical technique for applications where isotopic selectivity is of prime concern $[1,2]$. RIMS has the ability to eliminate isobaric interferences and hence promises to be an ideal technique for isotope ratio measurements. Such isotope ratio measurements are feasible only if the laser can indiscriminately ionize all the isotopes with equal efficiency. For all such applications, it appears logical to employ a laser with large linewidth $\left(\gamma_{L}\right)$ in comparison to the spectral width of the transition. Fairbank et al. [3] have observed an anomaly of about $\sim 31 \%$ in the odd-to-even response for tin and $\sim 15 \%$ for molybdenum when broadband lasers are used $[4,5]$. Such anomaly is not expected since the linewidth of the excitation laser is twice the frequency spread of the transition. The reason for such anomaly was traced to hyperfine coupling of the odd isotopes in two-step ionization [6-8]. Near-resonance ionization Mass Spectrometry (NRIMS) has been suggested by Brandon et al. for eliminating such anomalies [9]. NRIMS is based on the principle that, if ionization occurs on a time scale shorter than the hyperfine precession period, then the measured response will not be altered by the hyperfine coupling, and hence the isotope ratios measured using such a method should in principle represent the true abundances in the sample. Though the theoretical studies described above indicate the method for avoiding isotope-induced biases, they do not adequately describe the source of such anomaly. Xiong et al. [10] have studied the effect of laser intensity on the odd-even isotope response in the RIMS of tin. Apart from this work, only the work of Hasegawa and Suzuki [11] attributes the odd-even ratio is sensitive to the exciting-laser wavelength even when the laser bandwidth is broad. 
In the present paper, an alternative approach for understanding the anomaly in the odd to even isotope ratios of tin isotopes for relatively broadband lasers has been discussed. Using such an approach, it is ascertained that the inaccuracy in the peak frequency of the excitation laser to be the source for such anomalies in the odd to even isotope response of tin isotopes.

\section{Theoretical Approach}

Tin has ten isotopes having relative abundances of ${ }^{112} \mathrm{Sn}$ $(0.97 \%),{ }^{114} \mathrm{Sn}(0.65 \%),{ }^{115} \mathrm{Sn}(0.36 \%),{ }^{116} \mathrm{Sn}(14.53 \%),{ }^{117} \mathrm{Sn}$ $(7.38 \%),{ }^{118} \mathrm{Sn}(24.22 \%),{ }^{119} \mathrm{Sn}(8.58 \%),{ }^{120} \mathrm{Sn}(32.59 \%)$, ${ }^{122} \mathrm{Sn}(4.63 \%)$, and ${ }^{124} \mathrm{Sn}(5.79 \%)$. All the three odd isotopes have a nuclear spin of $1 / 2$. For this study, I have considered the $5 \mathrm{p}^{2}\left({ }^{3} \mathrm{P}_{0}\right)-5 \mathrm{p} 6 \mathrm{~s}\left({ }^{3} \mathrm{P}_{1}^{0}\right)(286.3317 \mathrm{~nm})$ transition of tin for which isotope shifts and hyperfine structure are well studied [12,13] (Table 1).

The theoretical approach adopted for the isotopic selectivity calculations is the spectral simulation (SS) method developed by our group [14-17]. In this approach the atomic line profile is generated assuming a Lorentzian profile taking into account the appropriate abundances, isotope shifts of the constituent isotopes and hyperfine structure of the odd isotopes. The Lorentzian line profile can be expressed as

$$
\mathrm{I}(\mathbf{w})=\left[\frac{2}{\pi \Delta \omega_{0}}\right]\left\{\frac{1}{1+\left\{2\left[\omega_{0}-\omega\right] / \Delta \omega_{0}\right\}^{2}}\right\} .
$$

The excitation atomic line profile was generated by using the appropriate laser linewidth. The laser line profile is assumed to be Guassian. However, a Voigt profile gives the most reliable results. The laser linewidth and the laser line shape are incorporated in the computation. Further details of this theoretical model have been discussed in our previous publications, and hence it is not elaborated here [14-17].

Isotopic selectivities are computed using the expression

$$
S(\omega)=\frac{I_{\mathrm{is} 1}(\omega)}{I_{\mathrm{all}}(\omega)},
$$

where $I_{\text {is } 1}(\omega)$ is the intensity of the isotope-1, and $I_{\text {all }}(\omega)$ is the intensity of all the isotopes. The abundances of the constituent isotopes can be calculated using the computed isotopic selectivity values. The SS approach has the following merits.

(a) The atomic lines are predominantly Guassian at peaks and Lorentzian at wings. Since the isotopic selectivities are limited by wing absorption of the atomic lines, it is adequate to consider the atomic profile to be Lorentzian.

(b) When the laser profile is assumed to be Lorentzian, then it may lead to unphysical results. This necessitates the introduction of a cutoff parameter $(\beta)$ in order to obtain realistic values. Assuming the laser line profile to be Guassian eliminates the need for the introduction of a cutoff parameter for computation. However, most reliable results can be obtained only when the computations are done using the laser line shape as determined during individual experiments. (c) The effect of hyperfine structure of the odd isotopes, abundance of the constituent isotopes, laser linewidth and laser line shape is incorporated in the computation.

(d) Since the isotope selectivity calculations do not include power broadening and Stark broadening effects, SS computations for the optimum laser intensity region should result in good approximation of the more comprehensive density matrix approach.

The isotopic selectivities thus obtained are used for the computation of abundance as a function of laser detuning $\left(\delta_{L}\right)$. The parameter $\beta$ is used as a measure of the difference in the odd to even isotope response for having a meaningful comparison of this work with the experimental results of Fairbank et al. [3] and Brandon et al. [9] as well as the experimental work carried out in this study:

$$
\beta=\frac{2\left[\mathbf{R}_{\text {odd }}-\mathbf{R}_{\text {even }}\right]}{\left[\mathbf{R}_{\text {odd }}+\mathbf{R}_{\text {even }}\right]} .
$$

The response $R(\omega)$ is defined as the ratio of the obtained abundance to the true abundance.

$$
\text { Response } R(\omega)=\frac{\text { Obtained Abundance }}{\text { True Abundance }} \text {. }
$$

\section{Experimental}

The experimental setup (Figure 1) used for the present investigations constitutes an $\mathrm{XeCl}$ excimer laser (Lumonics PM$848,300 \mathrm{~mJ}, 200 \mathrm{~Hz}, 15 \mathrm{~ns}$ ) pumping a grazing incidence dye laser (Lumonics HD-540, linewidth $\sim 1200 \mathrm{MHz}$ ) with rhodamine-6 G dye. A frequency doubler (Lumonics HT$1000, \mathrm{KDP}$ ) was used with the dye laser to obtain UV output for the ionization step. Calibration of the dye laser was done by recording the optogalvanic emission spectrum of the $\mathrm{Ne}$ lines in a commercial Au/Ne hollow cathode lamp (M/s. Cathodeon, UK) filled with neon buffer gas.

Water-cooled resistively heated atomic beam furnace with graphite crucible atomizer, similar to that used by Bekov and Letokhov [18], was used for generating the atomic beam. Tin pellets were loaded in the crucible and heated to about $\sim 1400^{\circ} \mathrm{C}$ by applying a current of $\sim 180 \mathrm{~A}$ using a power supply (20 V AC, $300 \mathrm{~A})$. The power supply was operated in constant current mode. A single-colour optical pyrometer was used for the calibration of the furnace temperature.

The dye laser output was frequency doubled using a frequency doubler (Lumonics HT_-1000, KDP) and tuned to $286.3317 \mathrm{~nm}$ for the excitation laser frequency, and another photon of the same wavelength was used for the ionization step. The pulse energy was $\sim 100 \mathrm{~mJ}$ to obtain multiphoton $(1+1)$ resonant ionization. The linewidth of the excitation laser is $1200 \mathrm{MHz}$. The atomic beam interacts with the laser beam orthogonally in the anticollinear geometry. The beam diameter was about $\sim 1.5 \mathrm{~mm}$ at the laser beamatomic beam interaction zone. At the furnace operating temperatures of $\sim 1400^{\circ} \mathrm{C}$, the residual Doppler width of the atomic beam as a result of collimation is $\sim 560 \mathrm{MHz}$. For some of the experiments, the furnace was operated at 


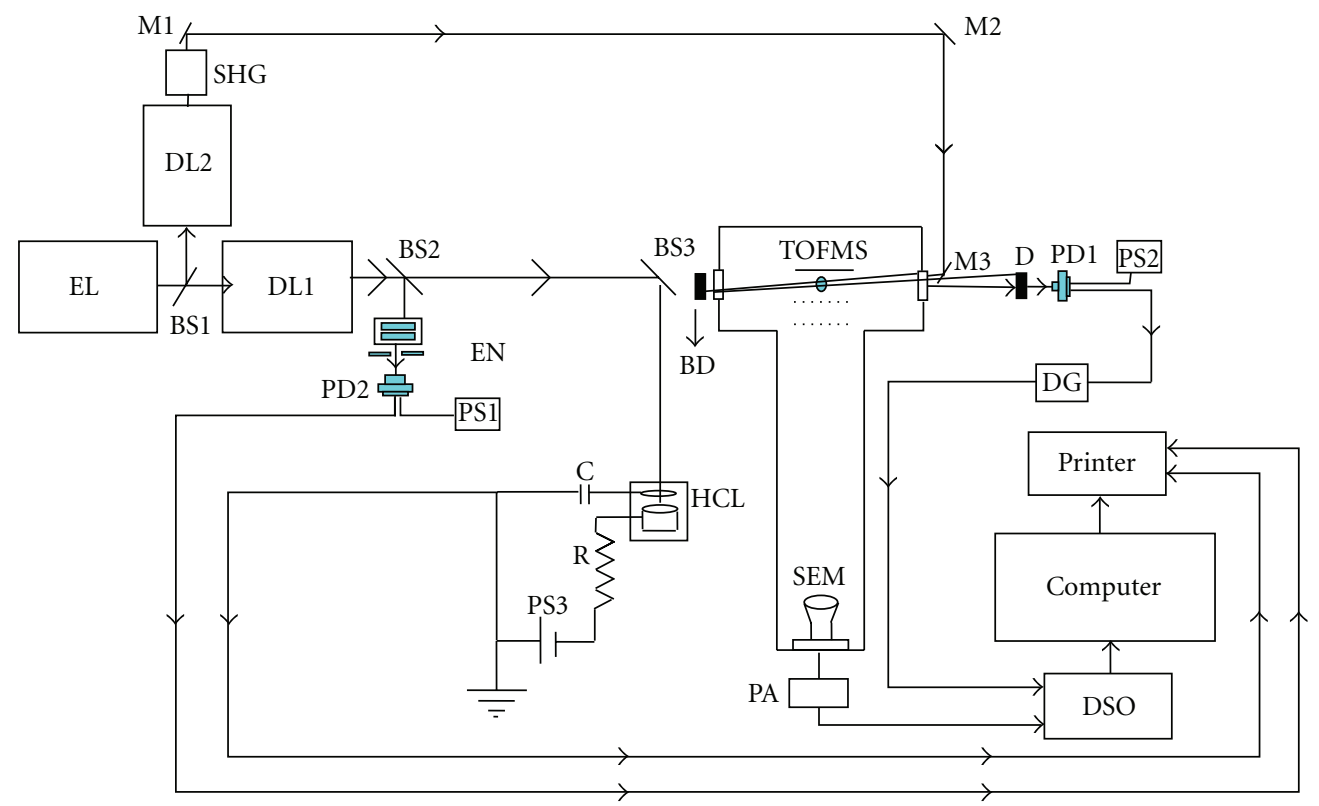

FIGURE 1: Schematic of the experimental setup of a resonance ionisation mass spectrometry (RIMS) system. BS: beam splitter, BD: beam dump, C: capacitor, D: diffusor, DG: delay generator, DL: dye laser, DSO: digital storage oscilloscope, EL: excimer laser, EN: 7.5GHz air spaced etalon, HCL: hollow cathode lamp, M: mirror, PA: preamplifier, PD: photo diode, PS: power supply, R: current limiting resistor, SEM: secondary electron multiplier, SHG: frequency doubler, TOFMS: time-of-flight mass spectrometer.

different temperatures to study the effect of varying Doppler and residual Doppler widths. Similarly, experiments have been performed at higher, laser linewidths $(2400 \mathrm{MHz})$.

An indigenously built Wiley-McLaren type of timeof-flight (TOF) mass spectrometer with a $120 \mathrm{~cm}$ long field free region and a resolution $(\mathrm{T} / 2 \Delta \mathrm{T})$ of 400 was used for recording the TOF spectrum of the photoions. The photoions were extracted into the mass spectrometer using two-stage acceleration. The total acceleration voltage applied was $2500 \mathrm{~V}$. A two-stage DC-300 MHz preamplifier (Stanford Research systems, SR 240) with single-stage gain of 5 was used to amplify the output of a secondary electron multiplier (Phillips, X959BL). The amplified signal was fed to a digital storage oscilloscope (Tektronix TDS-350, $200 \mathrm{MHz}$, $1 \mathrm{GS} / \mathrm{s}$ ) and stored. The data were averaged for 256 pulses and transferred to a computer for further analysis.

\section{Results and Discussion}

The response of the $\mathrm{Sn}$ isotopes is plotted as a function of laser detuning in Figure 2 for the $5 \mathrm{p}^{2}\left({ }^{3} \mathrm{P}_{0}\right)-5 \mathrm{p} 6 \mathrm{~s}\left({ }^{3} \mathrm{P}_{1}^{0}\right)$ $(286.3317 \mathrm{~nm})$ transition. It can be immediately realized that the response is critically dependent on the detuning frequency of the excitation laser. For example, when the excitation laser is detuned to $-17 \mathrm{GHz}$ (relative to the resonance position of ${ }^{120} \mathrm{Sn}$ isotope) the response of odd isotopes ${ }^{115} \mathrm{Sn},{ }^{117} \mathrm{Sn}$, and ${ }^{119} \mathrm{Sn}$ can be as large as 1.34 , 1.29 and 1.23 , respectively. In comparison to this, the even isotopes such as ${ }^{118} \mathrm{Sn},{ }^{120} \mathrm{Sn},{ }^{122} \mathrm{Sn}$, and ${ }^{124} \mathrm{Sn}$ have smaller responses $(0.95,0.90,0.85$, and 0.82 , resp.). It can also be observed from Figure 2 that, if the laser is detuned to the lower-frequency region, the response of the odd isotopes has the order ${ }^{115} \mathrm{Sn}>{ }^{117} \mathrm{Sn}>{ }^{119} \mathrm{Sn}$. On the other hand, the response of the odd isotopes is reversed when the frequency is detuned towards the higher frequency region $\left({ }^{119} \mathrm{Sn}>{ }^{117} \mathrm{Sn}>{ }^{115} \mathrm{Sn}\right)$. Such a trend, namely, the response of ${ }^{115} \mathrm{Sn}>>^{117} \mathrm{Sn}>>^{119} \mathrm{Sn}$ was in fact observed by Fairbank et al. [3] in sputter-initiated resonance ionization of tin isotopes which was inferred by them as not real and presumed to be an artifact due to insufficient correction for the count rate. This is in fact arising as a result of inaccuracy in tuning the excitation laser to the resonance. Computation of anomalies as a function of laser detuning has been carried out for a laser linewidth of $12 \mathrm{GHz}$ in order to compare the present results with Fairbank et al. [3]. A good agreement within the experimental errors has been obtained between these theoretical anomalies and the experimental results (Figure 3). Hence it can be concluded that the anomalies in tin isotopes are largely due to the inherent problem of tuning the laser to the exact resonance. In such cases, it is desirable to know the anomaly as a function of laser detuning so as to predict the detuning position where the anomaly could be minimized.

In order to find such detuned position, anomalies have been computed as a function of laser detuning and plotted in Figure 4 along with the measured anomalies by Brandon et al. [9]. The anomalies computed were in agreement with the experimentally determined anomalies particularly at smaller detuning $\left(\delta_{L} \approx \gamma_{L}\right)$. It can be seen from Figure 4 that an anomaly in the odd to even response can be as large as $\sim 0.31$ when the inaccuracy in the laser peak frequency is $\sim \pm 17 \mathrm{GHz}$. However, if the laser can be tuned to the exact resonance, the anomaly can be reduced to as small as -0.03 which is significantly smaller than 0.31 . At larger detuning 


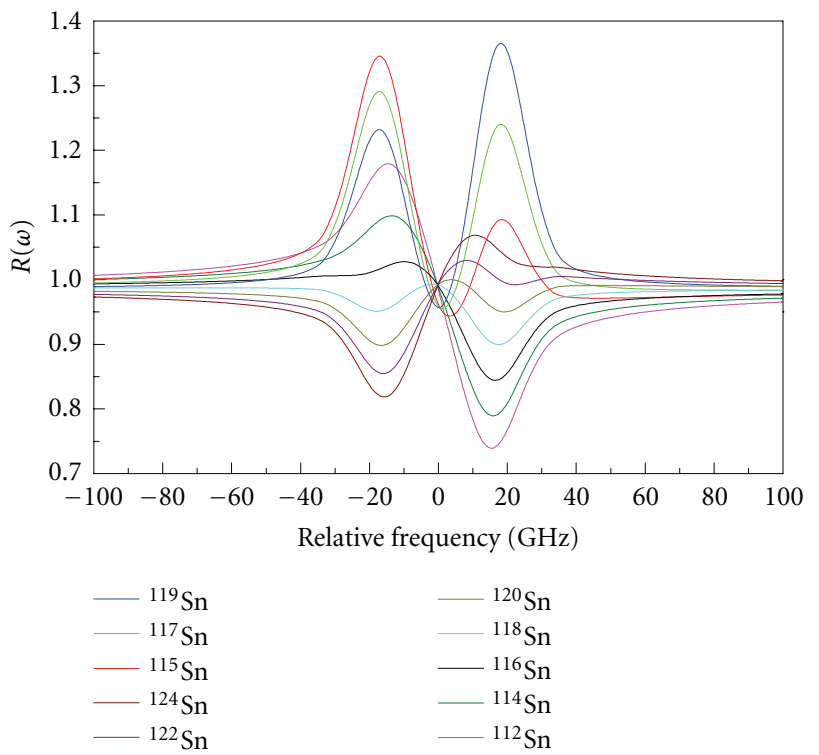

FIgURE 2: Response of the $S n$ isotopes as a function of laser detuning for the $5 \mathrm{p}^{2}{ }^{3} \mathrm{P}_{0}-5 \mathrm{p} 6 \mathrm{~s}\left({ }^{3} \mathrm{P}_{1}^{0}\right)(286.3317 \mathrm{~nm})$ transition for a laser linewidth of $15 \mathrm{GHz}$ and a Doppler width of $2 \mathrm{GHz}$.

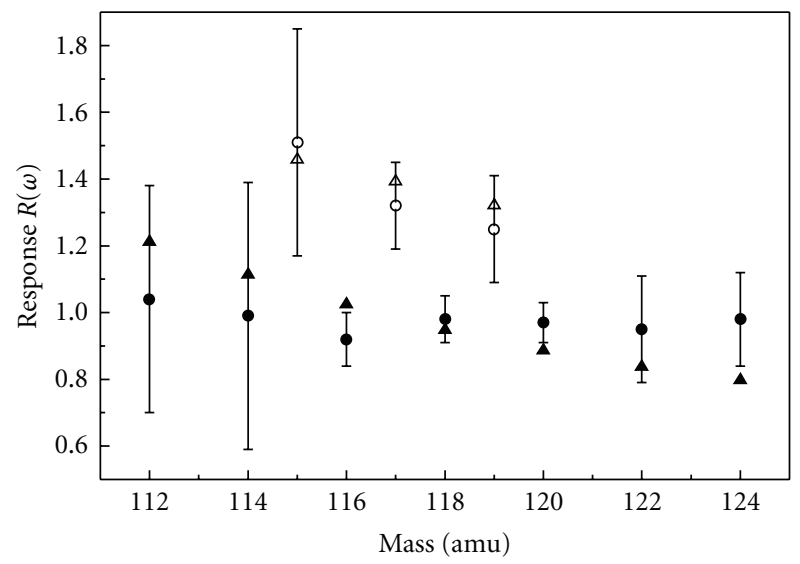

Circle (open): response of the odd isotopes (experimental) Circle (solid): response of the even isotopes (experimental) Up triangle (open): response of the odd isotopes (theoretical) Up triangle (solid): response of the even isotopes (theoretical) $\beta($ experimental $)=0.31(11), \beta$ (theoretical $)=0.35$ Open and close circle data taken from [3]

Figure 3: Response of the tin isotopes for the $5 p^{2}\left({ }^{3} \mathrm{P}_{0}\right)-5 \mathrm{p} 6 \mathrm{~s}$ $\left({ }^{3} \mathrm{P}_{1}^{0}\right)(286.3317 \mathrm{~nm})$ transition computed for an excitation laser linewidth of $12 \mathrm{GHz}$.

$\left(\delta_{L} \geq 2 \gamma_{L}\right)$, the experimentally determined anomaly was found to be larger than the theoretically predicted values. The increase in uncertainty with decreasing signal results in rather irreproducible anomalies, which could be the source of such difference. Moreover, in the experimental work carried out by Brandon et al. [9], at large detuning, the laser intensity was increased to widen the power-broadened width. The effect of power broadening at large detuning is

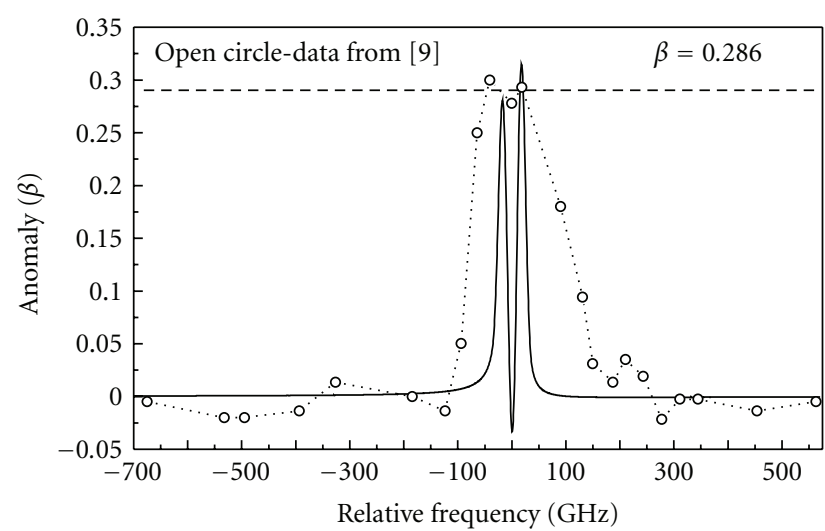

Figure 4: Anomaly $(\beta)$ of tin isotopes for the $5 p^{2}\left({ }^{3} \mathrm{P}_{0}\right)-5 p$ s $\left({ }^{3} \mathrm{P}_{1}^{0}\right)(286.3317 \mathrm{~nm})$ transition for a laser linewidth of $15 \mathrm{GHz}$ and a Doppler width of $2 \mathrm{GHz}$.

not incorporated in the present calculations and hence the difference in the present values and the experimental results is evident. The strong wavelength-dependent anomalies demand accurate positioning of the peak frequency of the excitation laser. For example, the anomaly $(\beta)$ can be contained within 0.01 when the accuracy in the frequency of $\pm 4 \mathrm{GHz}$ is ensured (Table 2). Similarly, for an anomaly $(\beta) \leq 0.05$, the accuracy in the positioning of the excitation laser should be within $\pm 5.5 \mathrm{GHz}$. When a broadband excitation laser $\left(\gamma_{L}=12-15 \mathrm{GHz}\right)$ is used, one linewidth of inaccuracy in the excitation laser can cause anomalies as large as 0.31 (Figure 4) [3,9]. This limits the utilization of moderately broadband lasers for reducing biases because an excitation laser with a linewidth of $15 \mathrm{GHz}$ having an accuracy in the laser peak frequency of $\pm 7.5 \mathrm{GHz}$ is not experimentally viable, resulting in unavoidable anomalies. Another interesting feature to note is the dip in the anomaly obtained by Brandon et al. when the laser is tuned close to $0 \mathrm{GHz}$ (Figure 4). When the laser is tuned to the resonance the anomaly expected is about -0.03 . This is significantly smaller from the anomaly obtained experimentally $(\beta \approx$ $0.31)$. The wide variation in the theoretical and experimental anomalies can be attributed to the inaccuracy in the laser peak frequency. As mentioned earlier, such anomalies are critically dependent on the laser peak frequency, and a highly accurate measurement of the laser peak frequency is necessary to obtain the theoretically predicted anomaly of -0.03 . Such a dip in the anomaly at the exact resonance has not been observed by Brandon et al. [9] owing to the inherent problem associated with the broadband lasers which limits the accurate determination of the laser peak frequency.

Since the anomaly varies drastically as a function of linewidth, two excitation lasers have been considered, one with a very large linewidth $\left(\gamma_{L}=60 \mathrm{GHz}\right)$ and the other with a relatively narrow linewidth $\left(\gamma_{L}=1.2 \mathrm{GHz}\right)$. With a very large increase in the laser linewidth ( eight to ten times the frequency spread of the isotopes), the anomaly in the odd to even isotope ratios reduces as expected due to complete overlap of the spectrum despite inaccuracy in the excitation laser wavelength. For example, when an excitation 
TABLE 1: The relative intensities and the relative frequency positions of tin isotopes for $5 \mathrm{p}^{2}\left({ }^{3} \mathrm{P}_{0}\right)-5 \mathrm{p} 6 \mathrm{~s}\left({ }^{3} \mathrm{P}_{1}^{0}\right)(286.3317 \mathrm{~nm})$ transition.

\begin{tabular}{lccc}
\hline Relative frequency $(\mathrm{MHz})$ & Isotope & Hyperfine component & Relative intensity $^{\#}$ \\
\hline-3002 & ${ }^{115} \mathrm{Sn}$ & $1 / 2-3 / 2$ & 0.7 \\
-2864 & ${ }^{117} \mathrm{Sn}$ & $1 / 2-3 / 2$ & 15.7 \\
-2684 & ${ }^{119} \mathrm{Sn}$ & $1 / 2-3 / 2$ & 17.5 \\
-1218 & ${ }^{112} \mathrm{Sn}$ & $0-1$ & 3.0 \\
-901 & ${ }^{114} \mathrm{Sn}$ & $0-1$ & 2.0 \\
-576 & ${ }^{116} \mathrm{Sn}$ & $0-1$ & 44.6 \\
-270 & ${ }^{118} \mathrm{Sn}$ & $0-1$ & 74.3 \\
0 & ${ }^{120} \mathrm{Sn}$ & $0-1$ & 100.0 \\
235 & ${ }^{122} \mathrm{Sn}$ & $0-1$ & 14.2 \\
441 & ${ }^{124} \mathrm{Sn}$ & $0-1$ & 17.8 \\
3589 & ${ }^{115} \mathrm{Sn}$ & $1 / 2-1 / 2$ & 0.4 \\
4314 & ${ }^{117} \mathrm{Sn}$ & $1 / 2-1 / 2$ & 7.8 \\
4828 & ${ }^{119} \mathrm{Sn}$ & $1 / 2-1 / 2$ & 8.8 \\
\hline
\end{tabular}

\#-Normalized for the abundance.

TABLE 2: A table of theoretically computed percentage anomalies for various laser linewidths.

\begin{tabular}{|c|c|c|c|c|c|}
\hline \multirow{2}{*}{$\begin{array}{l}\text { Laser } \\
\text { linewidth } \\
(\mathrm{LLW})(\mathrm{GHz})\end{array}$} & \multirow{2}{*}{$\begin{array}{l}\text { \% Anomaly }(\beta) \\
@ \text { resonance } \\
(0 \mathrm{GHz})\end{array}$} & \multicolumn{2}{|c|}{$\begin{array}{l}\text { The permissible inaccuracy in the laser peak } \\
\text { frequency }(\mathrm{GHz}) \text { on either side of the peak to } \\
\text { contain the anomaly }(\beta) \text { to }<1 \%\end{array}$} & \multicolumn{2}{|c|}{$\begin{array}{l}\text { The permissible inaccuracy in the laser peak } \\
\text { frequency }(\mathrm{GHz}) \text { on either side of the peak to } \\
\text { contain the anomaly }(\beta) \text { to }<5 \%\end{array}$} \\
\hline & & $\begin{array}{l}\text { Inaccuracy in the laser } \\
\text { peak frequency to the } \\
\text { - ve side of the peak }\end{array}$ & $\begin{array}{l}\text { Inaccuracy in the laser } \\
\text { peak frequency to the } \\
\text { +ve side of the peak }\end{array}$ & $\begin{array}{l}\text { Inaccuracy in the laser } \\
\text { peak frequency to the } \\
\text {-ve side of the peak }\end{array}$ & $\begin{array}{l}\text { Inaccuracy in the laser } \\
\text { peak frequency to the } \\
\text { +ve side of the peak }\end{array}$ \\
\hline 1.2 & -5.5 & - & - & - & - \\
\hline 15 & -3.3 & -3.4 & 4.6 & -5.3 & 6.3 \\
\hline 30 & -0.8 & -5.6 & 8.7 & -13.7 & 16.5 \\
\hline 45 & -0.4 & -9.0 & 14.4 & -26.1 & 31.1 \\
\hline 60 & -0.3 & -13.6 & 21.2 & -42.4 & 51.2 \\
\hline
\end{tabular}

laser with $60 \mathrm{GHz}$ linewidth is used, the anomaly is as small as -0.003 at the resonance (Table 2). Since the laser is sufficiently broadband, for an inaccuracy in the excitation laser peak frequency of half a linewidth (i.e., $\delta_{L}=1 / 2 \gamma_{L}$ $=30 \mathrm{GHz}$ ), the anomaly is 0.02 . (Table 3 ). Similarly, when the inaccuracy is one linewidth (i.e., $\delta_{L}=\gamma_{L}=60 \mathrm{GHz}$ ), the anomaly is 0.06 which is significantly smaller than 0.31 as in the case of a laser with linewidth of $15 \mathrm{GHz}$ (Table 3). Thus, for a laser with a very large linewidth, the inaccuracy (which is inherent for a broadband laser) in the wavelength of the excitation laser does not significantly increase the anomaly.

Alternatively, a relatively narrow-band laser with linewidth of $\sim 1.2 \mathrm{GHz}$ can be considered for the excitation. Even though it may appear unsuitable to employ such a laser for reducing the isotope-induced biases, a $1.2 \mathrm{GHz}$ laser can be tuned with better accuracy than a broadband laser within the spectral width of the transition. Anomaly in the odd to even isotope ratios can be limited to $\leq-0.05$, when it is tuned to the exact resonance. The negative value of $\beta$ can be understood from the frequency spread of the tin isotopes. The frequency spread of the tin isotopes for this transition is $\sim 7800 \mathrm{MHz}$ (Table 1). The even isotopes are bunched towards the central region of the transition; tuning the laser to the exact resonance results in higher even isotope ionization in comparison to the odd isotopes, resulting in negative value of $\beta$. When the laser is detuned by one linewidth on either side (i.e., $\delta_{L}= \pm \gamma_{L}$ ), the odd isotopes are preferentially ionized in comparison to the even isotopes. An inaccuracy of about three to four times of $\gamma_{L}$ in the excitation laser peak frequency limits the anomalies to $\leq 0.05$. Since this demand in the accuracy is experimentally viable, the anomalies could be significantly reduced.

In order to study in detail, the effect of other parameters on the odd-even isotope response further theoretical computations and simultaneous experimental verification were carried out. The effect of the mode structure of the laser on the isotopic selectivity has also been theoretically studied to determine the abundance values after incorporating the effect of the laser mode structure (Table 4). Similar theoretical computations were done for various laser linewidths (Tables 5 and 6). It has been found that the abundances computed with and without the incorporation of laser mode structure effects are not very different. This 
TABLE 3: A table of theoretically computed percentage anomalies for an inaccuracy of $0.5 \delta_{L}$ and $1.0 \delta_{L}$ for the various laser linewidths $\left(\delta_{L}\right)$.

\begin{tabular}{|c|c|c|c|c|c|}
\hline \multirow{2}{*}{$\begin{array}{l}\text { Laser } \\
\text { linewidth } \\
(\mathrm{LLW})(\mathrm{GHz})\end{array}$} & \multicolumn{2}{|c|}{$\begin{array}{l}\% \text { Anomaly }(\beta) \text { for an inaccuracy of } \\
0.5 \text { LLW on either side of the peak } \\
\text { frequency }\end{array}$} & \multicolumn{2}{|c|}{$\begin{array}{l}\% \text { Anomaly }(\beta) \text { for an inaccuracy of } \\
1.0 \text { LLW on either side of the peak } \\
\text { frequency }\end{array}$} & \multirow{2}{*}{$\begin{array}{l}\text { \% Anomaly }(\beta) @ \\
\text { resonance } \\
(0 \mathrm{GHz})\end{array}$} \\
\hline & $\begin{array}{l}\text { Inaccuracy in the laser } \\
\text { peak frequency of } 0.5 \mathrm{llw} \\
\text { to the +ve side of the } \\
\text { peak }\end{array}$ & $\begin{array}{l}\text { Inaccuracy in the laser } \\
\text { peak frequency of } 0.5 \mathrm{llw} \\
\text { to the - ve side of the } \\
\text { peak }\end{array}$ & $\begin{array}{l}\text { Inaccuracy in the laser } \\
\text { peak frequency of } 1.0 \mathrm{llw} \\
\text { to the +ve side of the } \\
\text { peak }\end{array}$ & $\begin{array}{l}\text { Inaccuracy in the laser } \\
\text { peak frequency of } 1.0 \mathrm{llw} \\
\text { to the - ve side of the } \\
\text { peak }\end{array}$ & \\
\hline 1.2 & -5.63 & -4.92 & -5.35 & -3.94 & -5.5 \\
\hline 15 & 10.77 & 8.33 & 29.38 & 27.16 & -3.3 \\
\hline 30 & 4.09 & 5.82 & 12.81 & 13.51 & -0.8 \\
\hline 45 & 2.67 & 4.00 & 7.95 & 8.97 & -0.4 \\
\hline 60 & 2.02 & 3.08 & 5.75 & 6.74 & -0.3 \\
\hline
\end{tabular}

TABLE 4: A table of theoretically computed isotopic selectivity and abundance values after incorporating laser mode structure effects of a laser with a linewidth of $2400 \mathrm{MHz}$.

\begin{tabular}{|c|c|c|c|c|c|c|c|}
\hline \multicolumn{8}{|c|}{ Isotopic selectivity values incorporating laser mode structure effects for a laser with a linewidth of $2400 \mathrm{MHz}$} \\
\hline \multicolumn{8}{|c|}{ Frequency of the laser mode structure $(\mathrm{MHz})$} \\
\hline \multirow{3}{*}{ Isotope } & 0 & -750 & -750 & -1500 & 1500 & \multirow{3}{*}{ Selectivity } & \multirow{3}{*}{$\begin{array}{l}\text { Abundance after } \\
\text { incorporating laser } \\
\text { mode structure } \\
\text { effects }\end{array}$} \\
\hline & $100 \%$ & $88.66 \%$ & $88.66 \%$ & $61.79 \%$ & $61.79 \%$ & & \\
\hline & $\begin{array}{c}\text { Central } \\
\text { mode }\end{array}$ & $\begin{array}{l}\text { First order mode } \\
\text { (LHS) }\end{array}$ & $\begin{array}{l}\text { First order mode } \\
\text { (RHS) }\end{array}$ & $\begin{array}{l}\text { Second order mode } \\
\text { (LHS) }\end{array}$ & $\begin{array}{l}\text { Second order mode } \\
\text { (RHS) }\end{array}$ & & \\
\hline${ }^{112} \mathrm{Sn}$ & 0.0047 & 0.0058 & 0.0039 & 0.0068 & 0.0033 & 0.016 & 0.934 \\
\hline${ }^{114} \mathrm{Sn}$ & 0.0034 & 0.0039 & 0.0030 & 0.0043 & 0.0026 & 0.011 & 0.664 \\
\hline${ }^{115} \mathrm{Sn}$ & 0.0008 & 0.0010 & 0.0008 & 0.0016 & 0.0011 & 0.003 & 0.184 \\
\hline${ }^{116} \mathrm{Sn}$ & 0.0815 & 0.0867 & 0.0748 & 0.0884 & 0.0682 & 0.260 & 15.55 \\
\hline${ }^{117} \mathrm{Sn}$ & 0.0155 & 0.0221 & 0.0146 & 0.0344 & 0.0196 & 0.062 & 3.73 \\
\hline${ }^{118} \mathrm{Sn}$ & 0.1408 & 0.1398 & 0.1377 & 0.1340 & 0.1315 & 0.442 & 26.68 \\
\hline${ }^{119} \mathrm{Sn}$ & 0.0179 & 0.0260 & 0.0156 & 0.0396 & 0.0193 & 0.070 & 4.20 \\
\hline${ }^{120} \mathrm{Sn}$ & 0.1921 & 0.1795 & 0.1991 & 0.1634 & 0.1991 & 0.604 & 36.41 \\
\hline${ }^{122} \mathrm{Sn}$ & 0.0273 & 0.0242 & 0.0298 & 0.0211 & 0.0311 & 0.086 & 5.19 \\
\hline \multirow[t]{2}{*}{${ }^{124} \mathrm{Sn}$} & 0.0337 & 0.0286 & 0.0385 & 0.0241 & 0.0418 & 0.1072 & 6.47 \\
\hline & & \multicolumn{3}{|c|}{ Total Selectivity } & & 1.66 & \\
\hline
\end{tabular}

is because of the large intensities used for the multi-photon process. The abundance of the tin isotopes has been plotted as a function of laser detuning for the $5 \mathrm{p}^{2}\left({ }^{3} \mathrm{P}_{0}\right)-5 \mathrm{p}$ 6s $\left({ }^{3} \mathrm{P}_{1}^{0}\right)(286.3317 \mathrm{~nm})$ transition for a laser linewidth of $1200 \mathrm{MHz}$ and a residual Doppler width of $400 \mathrm{MHz}$ (Figure 5). The abundances (Tables 4, 5, and 6) have also been experimentally and theoretically verified for the 1200 and $2400 \mathrm{MHz}$ laser linewidths (Figures 5, 6, 7, and 8). The theoretical and experimental abundances are in good agreement (Figures 5, 6, 7, and 8). The elaborate theoretical computations have been carried out to ensure and check the veracity of the simple spectral simulation approach that has been employed in this particular study. In this particular study, this theoretical model works because there is no coherent phenomenon to be considered for the computations. Similarly optical pumping also do not play a significant role during the excitation and hence the more comprehensive and thorough density matrix approach has been replaced with this simple SS approach. The experimental abundances have been calculated for various residual Doppler widths for a laser linewidth of $2400 \mathrm{MHz}$ (Figures 7 and 8). As inferred earlier, the anomaly is larger for the intermediate range of laser linewidths $(2400 \mathrm{MHz})$ as compared to the small $(\sim 1.2 \mathrm{GHz})$ or the very large laser linewidth $(60 \mathrm{GHz})$ cases. The abundances of the odd and even isotopes have been determined experimentally, and the anomaly $(\beta)$, thus obtained, has been tabulated as a function of laser frequency detuning in the collinear geometry-RIMS of tin isotopes (Table 7). As seen from Table 7, the anomaly increases as the detuning is increased on either side of zero detuning. But at very large detunings on the positive side the anomaly decreases as discussed in the previous sections. Percentage 
TABLE 5: A table of theoretically computed isotopic selectivity and abundance values after incorporating laser mode structure effects of a laser with a linewidth of $3600 \mathrm{MHz}$.

\begin{tabular}{|c|c|c|c|c|c|c|c|}
\hline \multicolumn{8}{|c|}{ Isotopic selectivity values incorporating laser mode structure effects of a laser linewidth of $3600 \mathrm{MHz}$} \\
\hline \multicolumn{8}{|c|}{ Frequency of the laser mode structure $(\mathrm{MHz})$} \\
\hline \multirow{3}{*}{ Isotope } & 0 & -750 & 750 & -1500 & 1500 & \multirow{3}{*}{ Selectivity } & \multirow{3}{*}{$\begin{array}{l}\text { Abundance after } \\
\text { incorporating laser } \\
\text { mode structure } \\
\text { effects }\end{array}$} \\
\hline & $100 \%$ & $88.66 \%$ & $88.66 \%$ & $61.79 \%$ & $61.79 \%$ & & \\
\hline & $\begin{array}{l}\text { Central } \\
\text { Mode }\end{array}$ & $\begin{array}{l}\text { First order mode } \\
\text { (LHS) }\end{array}$ & $\begin{array}{l}\text { First order mode } \\
\text { (RHS) }\end{array}$ & $\begin{array}{l}\text { Second order mode } \\
\text { (LHS) }\end{array}$ & $\begin{array}{l}\text { Second order mode } \\
\text { (RHS) }\end{array}$ & & \\
\hline${ }^{112} \mathrm{Sn}$ & 0.005 & 0.006 & 0.004 & 0.006 & 0.004 & 0.020 & 0.96 \\
\hline${ }^{114} \mathrm{Sn}$ & 0.004 & 0.004 & 0.003 & 0.004 & 0.003 & 0.014 & 0.66 \\
\hline${ }^{115} \mathrm{Sn}$ & 0.001 & 0.001 & 0.001 & 0.002 & 0.001 & 0.005 & 0.25 \\
\hline${ }^{116} \mathrm{Sn}$ & 0.081 & 0.083 & 0.076 & 0.085 & 0.072 & 0.319 & 15.26 \\
\hline${ }^{117} \mathrm{Sn}$ & 0.023 & 0.028 & 0.022 & 0.035 & 0.025 & 0.105 & 5.00 \\
\hline${ }^{118} \mathrm{Sn}$ & 0.137 & 0.136 & 0.136 & 0.134 & 0.132 & 0.542 & 25.90 \\
\hline${ }^{119} \mathrm{Sn}$ & 0.026 & 0.031 & 0.024 & 0.039 & 0.027 & 0.115 & 5.51 \\
\hline${ }^{120} \mathrm{Sn}$ & 0.186 & 0.179 & 0.191 & 0.169 & 0.191 & 0.736 & 35.18 \\
\hline${ }^{122} \mathrm{Sn}$ & 0.027 & 0.025 & 0.028 & 0.023 & 0.029 & 0.086 & 5.01 \\
\hline \multirow[t]{2}{*}{${ }^{124} \mathrm{Sn}$} & 0.033 & 0.030 & 0.036 & 0.027 & 0.038 & 0.131 & 6.26 \\
\hline & & \multicolumn{3}{|c|}{ Total selectivity } & & 2.092 & \\
\hline
\end{tabular}

TABLE 6: A table of theoretically computed isotopic selectivity and abundance values after incorporating laser mode structure effects of a laser with a linewidth of $4800 \mathrm{MHz}$.

\begin{tabular}{|c|c|c|c|c|c|c|c|}
\hline \multicolumn{8}{|c|}{ Isotopic selectivity values incorporating laser mode structure effects of a laser linewidth of $4800 \mathrm{MHz}$} \\
\hline \multicolumn{8}{|c|}{ Frequency of the laser mode structure $(\mathrm{MHz})$} \\
\hline \multirow{3}{*}{ Isotope } & 0 & -750 & 750 & -1500 & 1500 & \multirow{3}{*}{ Selectivity } & \multirow{3}{*}{$\begin{array}{l}\text { Abundance after } \\
\text { incorporating lases } \\
\text { mode structure } \\
\text { effects }\end{array}$} \\
\hline & $100 \%$ & $93.45 \%$ & $93.45 \%$ & $76.28 \%$ & $76.28 \%$ & & \\
\hline & $\begin{array}{l}\text { Central } \\
\text { mode }\end{array}$ & $\begin{array}{l}\text { First order mode } \\
\text { (LHS) }\end{array}$ & $\begin{array}{l}\text { First order mode } \\
\text { (RHS) }\end{array}$ & $\begin{array}{l}\text { Second order mode } \\
\text { (LHS) }\end{array}$ & $\begin{array}{l}\text { Second order mode } \\
\text { (RHS) }\end{array}$ & & \\
\hline${ }^{112} \mathrm{Sn}$ & 0.0050 & 0.0055 & 0.0046 & 0.0059 & 0.0042 & 0.0221 & 0.964 \\
\hline${ }^{114} \mathrm{Sn}$ & 0.0035 & 0.0037 & 0.0033 & 0.0038 & 0.0031 & 0.0152 & 0.662 \\
\hline${ }^{115} \mathrm{Sn}$ & 0.0014 & 0.0015 & 0.0014 & 0.0017 & 0.0014 & 0.0065 & 0.282 \\
\hline${ }^{116} \mathrm{Sn}$ & 0.0792 & 0.0811 & 0.0766 & 0.0822 & 0.0734 & 0.3452 & 15.054 \\
\hline${ }^{117} \mathrm{Sn}$ & 0.0285 & 0.0312 & 0.0280 & 0.0357 & 0.0296 & 0.1337 & 5.828 \\
\hline${ }^{118} \mathrm{Sn}$ & 0.1338 & 0.1334 & 0.1323 & 0.1317 & 0.1304 & 0.5824 & 25.394 \\
\hline${ }^{119} \mathrm{Sn}$ & 0.0314 & 0.0345 & 0.0302 & 0.0393 & 0.0326 & 0.1467 & 6.397 \\
\hline${ }^{120} \mathrm{Sn}$ & 0.1811 & 0.1764 & 0.1840 & 0.1702 & 0.1847 & 0.7886 & 34.389 \\
\hline${ }^{122} \mathrm{Sn}$ & 0.0258 & 0.0246 & 0.0267 & 0.0233 & 0.0273 & 0.1123 & 4.897 \\
\hline \multirow[t]{2}{*}{${ }^{124} \mathrm{Sn}$} & 0.0322 & 0.0304 & 0.0340 & 0.0280 & 0.0353 & 0.1406 & 6.132 \\
\hline & & \multicolumn{3}{|c|}{ Total selectivity } & & 2.29325 & \\
\hline
\end{tabular}

anomaly $(\beta)$ has been theoretically calculated, as a function of laser intensity in the anticollinear geometry of RIMS of tin for a laser linewidth of $15 \mathrm{GHz}$ and a residual Doppler width of $2 \mathrm{GHz}$. It is observed that in the lower intensity, and for the very high intensity range, the percentage anomalies are not very large as also inferred by Hasegawa and Suzuki [11]. The even mass isotopes and odd isotopes are ionized almost equally when the intensity is less. Since the excitation transition is not saturated the populations of the ground and the excited states do not become equalized. On the contrary the odd mass isotopes are ionized more in the intermediate intensity region of the laser. At intermediate intensity, the resonant transition is completely saturated, but the ionization transition is not. For the even isotopes $50 \%$ of the atoms are available for ionization when the excitation transition is saturated, whereas, $2 / 3$ of the odd isotopes are 


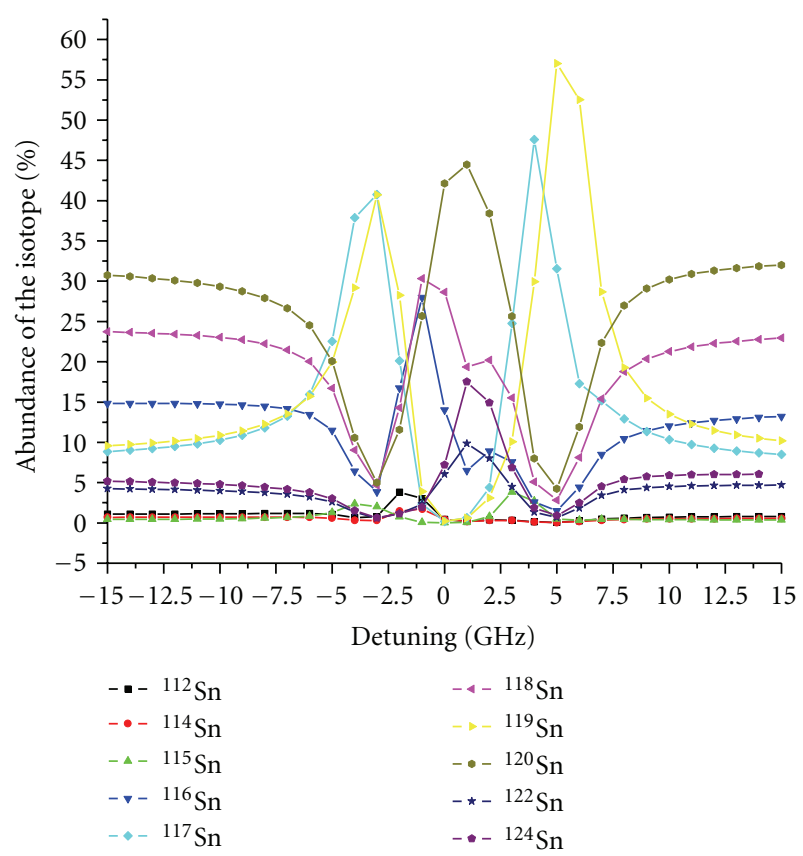

FIgUre 5: Experimental abundance of the tin isotopes as a function of laser detuning for the $286.3317 \mathrm{~nm}$ transition for a laser linewidth of $1200 \mathrm{MHz}$ and a residual Doppler width of $400 \mathrm{MHz}$.

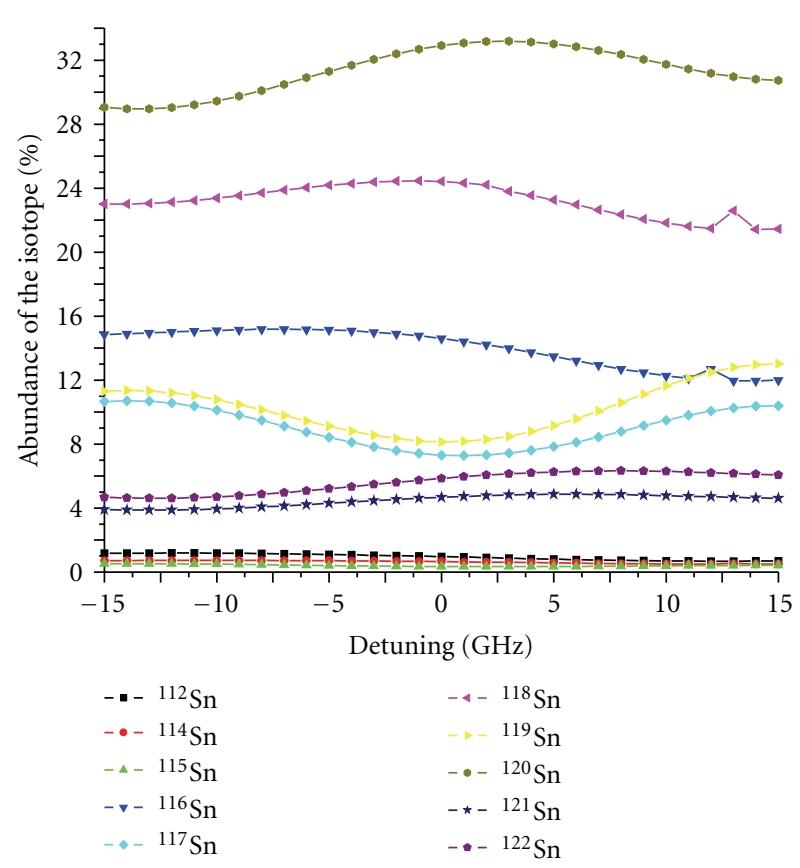

FIGURE 6: Experimental abundance of the tin isotopes as a function of laser detuning for $286.3317 \mathrm{~nm}$ transition for a laser linewidth of $1200 \mathrm{MHz}$ and a residual Doppler width of $2000 \mathrm{MHz}$.

available for ionization from the excited state. Therefore, a population effect with $4 / 3$ as much population available for the odd isotopes as the even isotopes, the anomaly $\beta$ amounts to $2(4 / 3-1) /(4 / 3+1)=0.29$. At high intensities, all the

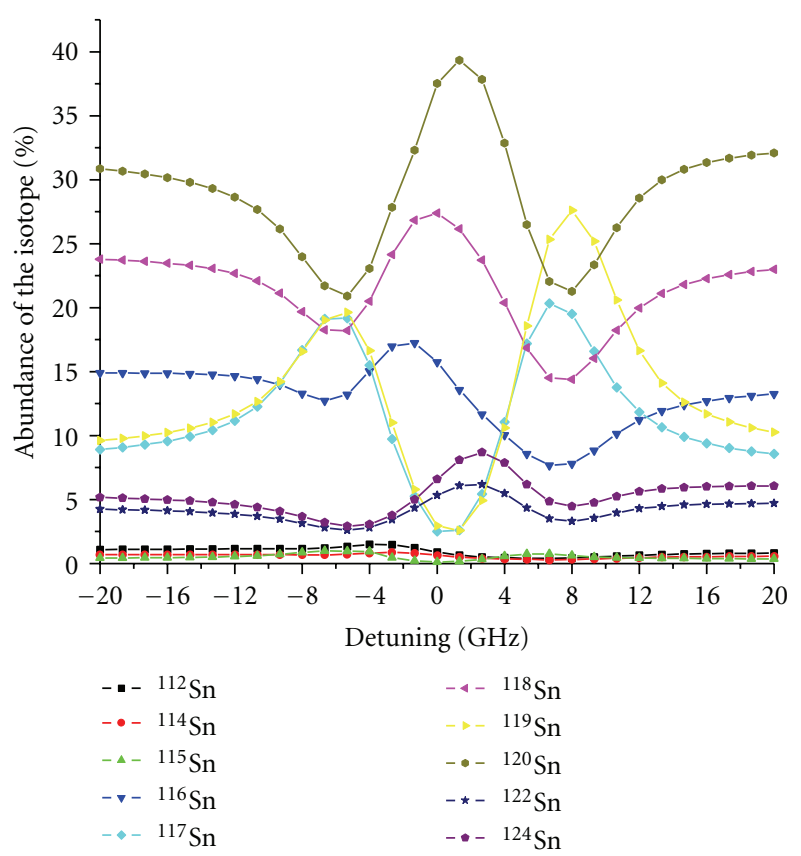

FIGURE 7: Experimental abundance of the tin isotopes as a function of laser detuning for $286.3317 \mathrm{~nm}$ transition for a laser linewidth of $2400 \mathrm{MHz}$ and a residual Doppler width of $400 \mathrm{MHz}$.

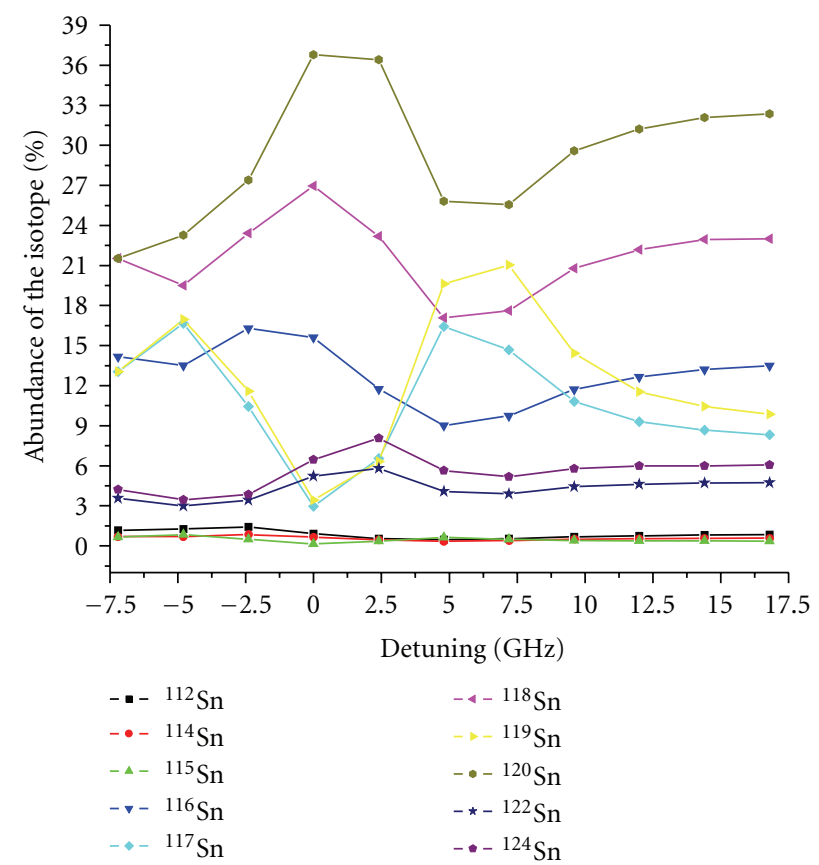

FIGURE 8: Experimental abundance of the tin isotopes as a function of laser detuning for the $286.3317 \mathrm{~nm}$ transition for a laser linewidth of $2400 \mathrm{MHz}$ and a residual Doppler width of $2800 \mathrm{MHz}$.

isotopes are indiscriminately ionized, and hence the oddeven response is the same. Moreover, at high intensities the bound state populations do not become equal, and hence the odd-even anomaly gets reduced. 
TABLE 7: A table of the experimentally determined abundances and responses of the odd and even isotopes. Experimentally determined anomaly $(\beta)$ as a function of laser frequency detuning for collinear-RIMS.

\begin{tabular}{|c|c|c|c|c|c|}
\hline $\begin{array}{l}\text { Detuning } \\
(\mathrm{GHz})\end{array}$ & $\begin{array}{l}\text { Abundance of odd } \\
\text { isotope }\end{array}$ & $\begin{array}{l}\text { Abundance of even } \\
\text { isotope }\end{array}$ & $\begin{array}{l}\text { Response of the odd isotope } \\
\mathrm{R}_{\text {odd }} \\
\end{array}$ & $\begin{array}{l}\text { Response of the even isotope } \\
\mathrm{R}_{\text {even }} \\
\end{array}$ & $\begin{array}{c}\text { Anomaly } \\
\beta\end{array}$ \\
\hline-7.2 & 17.16 & 26.72 & 2.00 & 0.78 & 0.88 \\
\hline-4.8 & 13.98 & 24.73 & 1.63 & 0.75 & 0.74 \\
\hline-2.4 & 10.55 & 28.68 & 1.23 & 0.87 & 0.34 \\
\hline 0 & 10.48 & 27.61 & 1.22 & 0.84 & 0.37 \\
\hline 2.4 & 12.58 & 23.51 & 1.47 & 0.71 & 0.69 \\
\hline 4.8 & 14.43 & 22.67 & 1.68 & 0.69 & 0.84 \\
\hline 7.2 & 14.49 & 23.18 & 1.69 & 0.70 & 0.83 \\
\hline 9.6 & 14.63 & 24.07 & 1.70 & 0.73 & 0.80 \\
\hline 12.0 & 11.73 & 29.99 & 1.37 & 0.91 & 0.40 \\
\hline 14.4 & 11.05 & 31.07 & 1.28 & 0.94 & 0.30 \\
\hline
\end{tabular}

TABLE 8: A table of the theoretically determined percentage anomaly $(\beta)$ as a function of laser intensity in the anticollinear-RIMS of tin isotopes for a laser linewidth of $15 \mathrm{GHz}$ and a residual Doppler width of $2 \mathrm{GHz}$.

\begin{tabular}{|c|c|c|c|c|c|}
\hline \multirow{2}{*}{ Isotope } & \multicolumn{5}{|c|}{ Intensity of the laser $\left(\mathrm{W} / \mathrm{cm}^{2}\right)$} \\
\hline & $5 \times 10^{6}$ & $1 \times 10^{7}$ & $2 \times 10^{8}$ & $1 \times 10^{9}$ & $5 \times 10^{9}$ \\
\hline${ }^{112} \mathrm{Sn}$ & 0.9961 & 0.747 & 1.193 & 0.835 & 1.137 \\
\hline${ }^{114} \mathrm{Sn}$ & 0.9992 & 0.798 & 1.110 & 0.877 & 1.087 \\
\hline${ }^{115} \mathrm{Sn}$ & 0.9721 & 1.090 & 1.353 & 0.981 & 1.156 \\
\hline${ }^{116} \mathrm{Sn}$ & 1.0017 & 0.855 & 1.033 & 0.921 & 1.038 \\
\hline${ }^{117} \mathrm{Sn}$ & 0.9691 & 1.232 & 1.297 & 1.047 & 1.117 \\
\hline${ }^{118} \mathrm{Sn}$ & 1.0034 & 0.914 & 0.965 & 0.965 & 0.993 \\
\hline${ }^{119} \mathrm{Sn}$ & 0.9674 & 1.352 & 1.238 & 1.101 & 1.081 \\
\hline${ }^{120} \mathrm{Sn}$ & 1.0045 & 0.970 & 0.100 & 1.005 & 0.954 \\
\hline${ }^{122} \mathrm{Sn}$ & 1.0051 & 1.022 & 0.865 & 1.041 & 0.922 \\
\hline${ }^{124} \mathrm{Sn}$ & 1.0053 & 1.070 & 0.828 & 1.074 & 0.895 \\
\hline Percentage anomaly & $-3.3 \%$ & $29.4 \%$ & $27 \%$ & $8.3 \%$ & $10.8 \%$ \\
\hline
\end{tabular}

\section{Conclusion}

The effects of laser parameters such as intensity, accuracy of the excitation laser wavelength, and bandwidth on the determination of the tin isotope ratio have been analyzed theoretically and experimentally. The anomalies in the odd to even isotope response for the $5 \mathrm{p}^{2}{ }^{3} \mathrm{P}_{0}-5 \mathrm{p} 6 \mathrm{~s}$ ${ }^{3} \mathrm{P}_{1}^{o}(286.3317 \mathrm{~nm})$ transition of tin have been theoretically studied using spectral simulation approach as well as experimentally. Anomalies as large as $31 \%$ can arise due to inaccuracy in the excitation laser frequency. The source for such anomalies was found to be the inaccuracy in the excitation laser wavelength for a moderately broadband laser. This requires highly accurate determination of the excitation laser frequency. However, for broadband lasers such accurate tuning is not experimentally viable resulting in unavoidable anomalies. The anomalies can be reduced by two alternative approaches. One approach is by way of using very large bandwidth lasers $(\sim 60 \mathrm{GHz})$. The other approach is by way of employing a relatively narrow-band laser $(\sim 1.2 \mathrm{GHz})$, where 3-4 times $\gamma_{L}$ inaccuracy in the laser peak frequency does not increase anomalies significantly. Hence, such lasers could have potential advantage over moderately broadband lasers.

Determination of isotope ratios using resonance ionization mass spectrometry has inherent disadvantage due to difference in the probability of ionization of odd and even isotopes. The other effects such as polarization selection rules induced biases and linewidth effects of the excitation lasers further complicate the isotope ratio measurements. However, a more deep understanding of the source of such biases helps in eliminating such biases. Even though the utilization of appropriate lasers can reduce the anomalies considerably, near-resonance ionization mass spectrometry seems to be the most universal method for avoiding such biases.

\section{Acknowledgment}

The author would like to thank Dr. M. V. Suryanarayana for his keen interest and support for the experimental and theoretical studies related to this project. 


\section{References}

[1] V. S. Letokhov, Laser Photoionisation Spectroscopy, Academic Press, Orlando, Fla, USA, 1987.

[2] G. S. Hurst and M. G. Payne, Principles and Applications of Resonance Ionization Spectroscopy, Adam Hilger, Bristol, UK, 1988.

[3] W. M. Fairbank, M. T. Spaar, J. E. Parks, and J. M. R. Hutchinson, "Anomalous odd- to even-mass isotope ratios in resonance ionization with broad-band lasers," Physical Review A, vol. 40, no. 4, pp. 2195-2198, 1989.

[4] W. M. Fairbank Jr., E. Riis, R. D. LaBalle, J. E. Parks, M. T. Spaar, and G. S. Hurst, "A Search for new elementary particles using sputter initiated resonance ionization spectroscopy," in Proceedings of the 3rd International Symposium on Resonance Ionization Spectroscopy and Its Applications, G. S. Hurst and C. Grey Morga, Eds., Institute of Physics Conference Series Number 84, pp. 275-280, The Institute of Physics, Wales, UK, September 1986.

[5] W. M. Fairbank Jr., M. T. Spaar, J. E. Parks, and J. M. R. Hutchinson, "Observation of anomolous isotope ratios in SIRIS measurements of molybdenum," in Proceedings of the 4th International Symposium on Resonance Ionization Spectroscopy and Its Applications, T. B. Lucatorto and J. E. Parks, Eds., Institute of Physics Conference Series 94, pp. 293-296, The Institute of Physics, Gaithersburg, Md, USA, April 1988.

[6] P. Lambropoulos and A. Lyras, "Theory of resonant ionization by broad-band radiation in the determination of isotopic abundances," Physical Review A, vol. 40, no. 4, pp. 2199-2202, 1989.

[7] A. Lyras, B. Zorman, and P. Lambropoulos, "Theory of doubly resonant ionization by broad-band radiation applied to the determination of isotopic abundances," Physical Review A, vol. 42, no. 1, pp. 543-549, 1990.

[8] M. G. Payne, S. L. Allman, and J. E. Parks, "Effect of hyperfine structure on ionization efficiencies in stepwise ionization using broad bandwidth lasers," Spectrochimica Acta Part B, vol. 46, no. 11, pp. 1439-1457, 1991.

[9] W. D. Brandon, W. R. Garrett, C. H. Chen, S. L. Allman, and M. G. Payne, "Experimental verification of a simple method to avoid isotopic biases resulting from hyperfine structure in resonant ionization mass spectroscopy," Spectrochimica Acta Part B, vol. 49, no. 11, pp. 1057-1066, 1994.

[10] X. Xiong, J. M. R. Hutchinson, J. Fassett, and W. M. Fairbank Jr., in Proceedings of the 6th International Symposium on Resonance Ionization Spectroscopy and Its Applications, J. D. Winefordner and I. M. Kolthoff, Eds., Institute Of Physics Conference Series 128, p. 157, John Wiley \& Sons, Santa Fe, NM, USA, May 1992.

[11] S. Hasegawa and A. Suzuki, "Effects of the laser parameters on the determination of the Sn isotope ratio," Physical Review A, vol. 48, no. 6, pp. 4588-4593, 1993.

[12] E. B. Saloman, "A resonance ionization spectroscopy/resonance ionization mass spectrometry data service. III-Data sheets for Sb, Bi, P, Na and Sn," Spectrochimica Acta Part B, vol. 47 , no. 4, pp. 517-543, 1992.

[13] M. Anselment, K. Bekk, A. Hanser et al., "Charge radii and moments of tin nuclei by laser spectroscopy," Physical Review C, vol. 34, no. 3, pp. 1052-1059, 1986.

[14] M. V. Suryanarayana and M. Sankari, "Simulation of isotopic selectivities and isotope ratio enhancement factors for gadolinium and lanthanum using narrow band laser excitation," Zeitschrift fur Physik D, vol. 39, no. 1, pp. 35-40, 1997.
[15] M. V. Suryanarayana and M. Sankari, "Comparison of density matrix and spectral simulation approaches for the calculation of isotopic selectivities," Journal of Quantitative Spectroscopy and Radiative Transfer, vol. 67, no. 1, pp. 65-77, 2000.

[16] M. V. Suryanarayana, M. Sankari, and S. Gangadharan, "Determination of $\mathrm{Li} / \mathrm{Li}$ isotope ratio using two photon resonance three photon resonance ionisation mass spectrometry," International Journal of Mass Spectrometry and Ion Processes, vol. 173, no. 3, pp. 177-189, 1998.

[17] M. Sankari and M. V. Suryanarayana, "Effect of Sr hyperfine structure on the isotope selective excitation of $\mathrm{Sr}$ and $\mathrm{Sr}$ isotopes in collinear resonance ionization spectroscopy," Journal of Physics B, vol. 35, no. 4, pp. 983-995, 2002.

[18] G. I. Bekov and V. S. Letokhov, "Laser atomic photoionization spectral analysis of element traces," Applied Physics B, vol. 30, no. 4, pp. 161-176, 1983. 

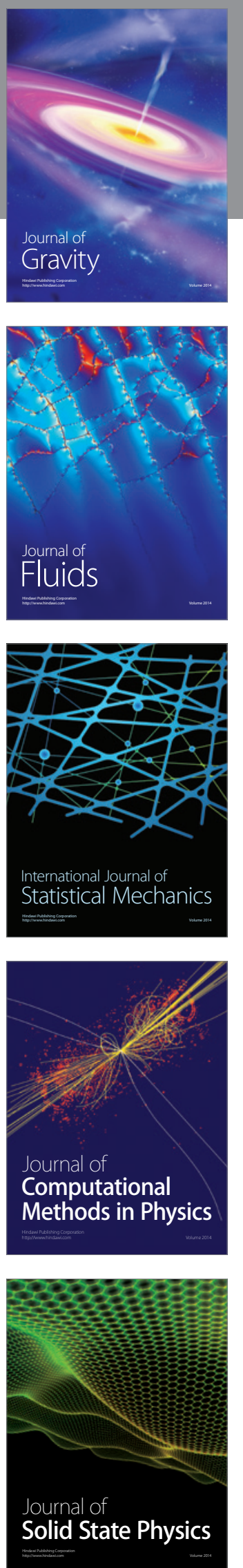

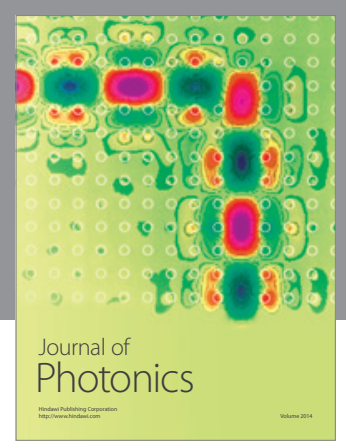

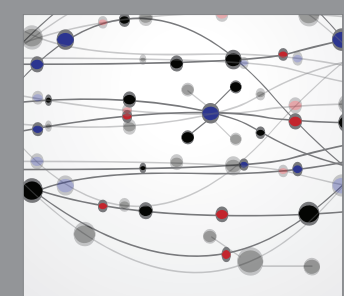

The Scientific World Journal
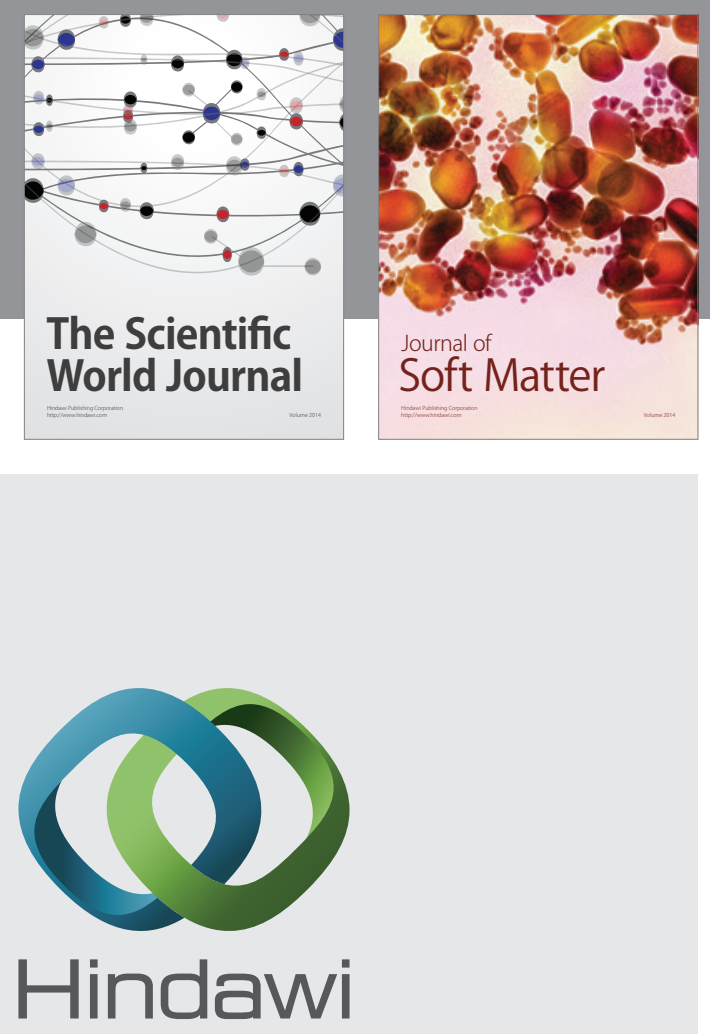

Submit your manuscripts at

http://www.hindawi.com
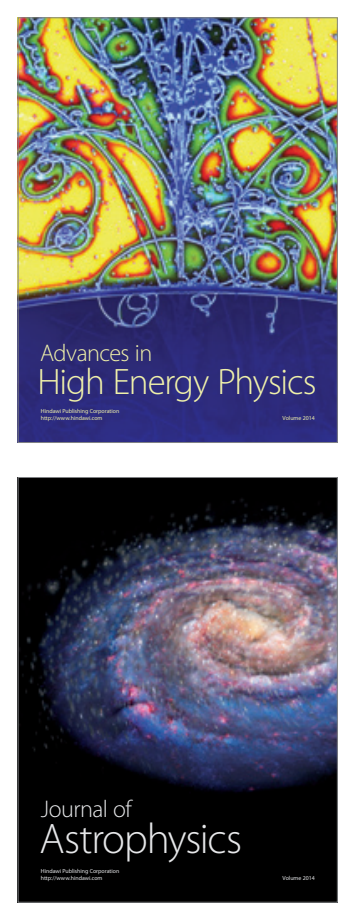
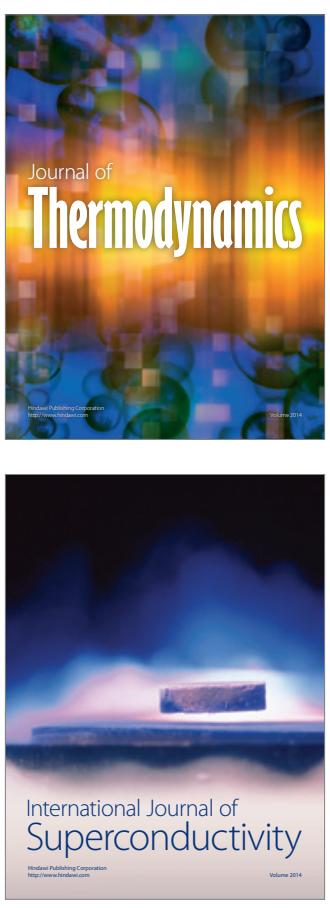
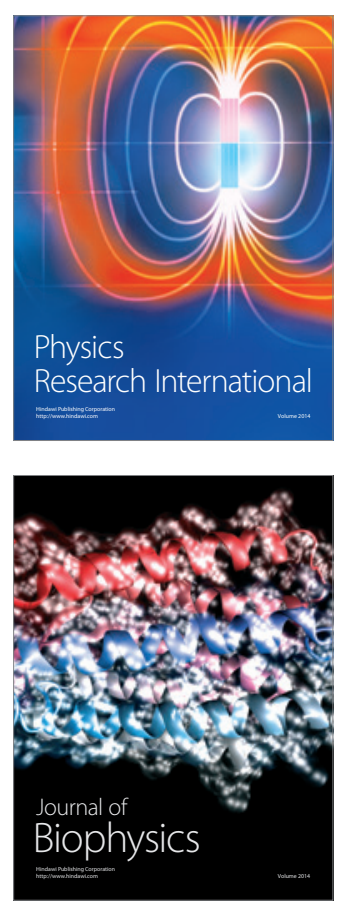
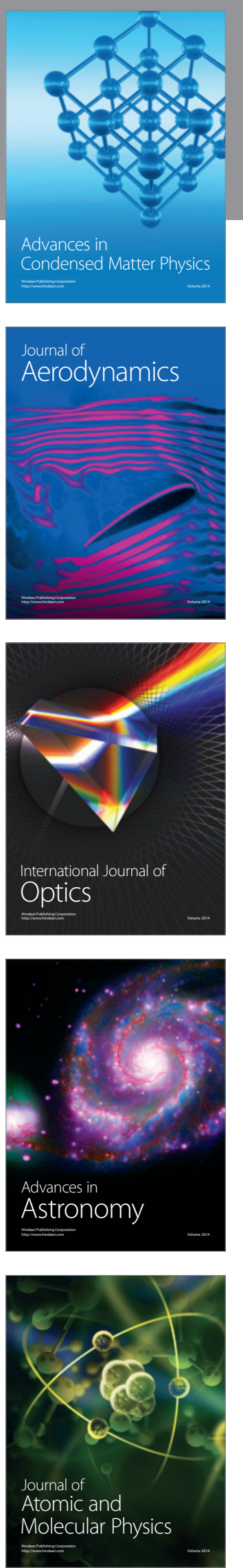